\title{
Development of Rational Rail Network Topology for High-Speed and Conventional Trains Based on Bacterial Foraging Optimization
}

\author{
Sergii Panchenko ${ }^{1}$, Tatyana Butko ${ }^{1}$, Andrii Prokhorchenko ${ }^{1 *}$, Larisa Parkhomenko ${ }^{1}$, Oleg Zhurba ${ }^{2}$ \\ ${ }^{1}$ Ukrainian State University of Railway Transport \\ ${ }^{2}$ Zhytomyr State Technological University \\ *Corresponding author E-mail: prokhorchenko@kart.edu.ua
}

\begin{abstract}
Development of projects on higher speeds of passenger trains on the world railways requires investigation into the balanced combination of routes for high-speed and conventional trains considering railway network topology. The objective of the study is consideration of railway network peculiarities regarding transportation demand by designing a mathematical model to find an optimal passenger train flow distribution. In order to formalize the process of a simultaneous search for the rail passenger network topology and determination of the most probable distribution within the formed train flow network for high-speed and conventional trains it has been proposed to use a criterion as a system entropy adapted to the rail passenger-oriented transportation. The concept of entropy is based on isomorphism in systems, which allows monitoring and implementing the link between a micro and macro level in the passenger transportation system. To solve the mathematical model the authors have used an optimization method based on Bacterial Foraging Optimization (BFO). The implementation of the mathematical model based on BFO will make it possible to theoretically substantiate the efficiency of existing and promising projects on higher speeds of passenger trains on the rail transport regarding adaptation of the network topology to transportation demand.
\end{abstract}

Keywords: Bacterial Foraging Optimization; high-speed train; network topology; passenger transportation planning; railway transport.

\section{Introduction}

Implementation of projects on higher speeds of passenger trains on the world railways requires investigation into a balanced coexistence of high-speed and conventional traffic with consideration of rail network topology development. The solution to the problem is rather complicated and based on synthesis of investigation into the theory of traffic flows, transport geography, network development and transport economic theory [1-3].

The latest achievements in the field of network design problems [4-6] confirmed that macro characteristics of a complicated transport system are not formed on the base of central top-down hierarchical planning, but determined by self-organizing passenger flows based on demand for transportation. Artificial demand stimulation methods for passenger trains of technical and economic parameters which do not meet the transportation requirements lead to re-distribution of flows along the line with possible refusals from some categories of passengers to travel, which finally results to the whole transport system unprofitability.

A great deal of investigation concerns a search for a rational distribution of passenger train routes and rail network topology formation. The work [7] proposing a double-stage optimization model for planning rail high-speed passenger transportation for Chinese rail network is an example of such research. In the framework of the research $[8,9]$ the authors made calculation regarding the selection of passenger train routes (calculation of passenger train plan formation). However, existing methods to find a rational rail network topology make it possible to only determine passen- ger flow distribution within the network regardless transportation demand from passengers of different income levels. Besides the research was conducted only for high-speed passenger transportation regardless the existence and development of the conventional passenger rail network which always influences the efficiency of high-speed transportation. The above mentioned testifies that there is a need to make a further research into formalization of procedures to search for a rational rail network topology for high-speed and conventional train traffic.

\section{Methodology Adopted}

In order to solve the problem, an entropy model to search for a rational passenger train flow distribution, which takes into account the rail network topology of high-speed and conventional traffic, has been formed. It is based on multi-agent optimization methods and demonstrates the performance of a rail passenger transportation system in terms of transport system development subject to demand for transportation.

The study proposes to use the concept of entropy because of isomorphism in systems for investigation into self-organization of train flow distribution; it allows researching and implementing connection between micro and macro levels in the passenger transportation system. On the base of the second law of thermodynamics, the rail passenger transportation system is presented as a closed physical system tending to a steady, equilibrium state, when uncertainty, measured as entropy, is maximal [10]. The maximum entropy in the system makes it possible to find such a 
system status, which is characterized by spatial distribution within a passenger flow network, and, as a consequence, high-speed and conventional train flows, which in terms of probability is reminiscent of one possible for a real transport system considering patterns of collective behavior.

\section{Mathematical Model to Design a Rational Rail Network Topology of High-Speed and Conventional Train Traffic}

In order to formalize the spatial structure of a rail passenger system the authors have used the undirected graph $G(P, E)$, where $P$ is the multiplicity of nodes corresponding to technical stations with the possibility to organize passenger train transportation between them $(i, j) \in P$, where $i=1, \ldots, p, j=1, \ldots, p ; E$ is the multiplicity of edges $e_{i j}$, corresponding to rail lines between passenger stations, $e_{i j} \in E$. Possible destinations of passenger trains are characterized by intensity of train flows of different types $x_{i j}^{k}, k=1, \ldots, K, x_{i j}^{k} \geq 0$, particularly $k=1$ is the high speed passenger train and $k=2$ is the conventional passenger train. In order to generate a rail network topology of different types the authors have proposed to use an incidence matrix with type elements.

$$
\xi_{i j}^{k}=\left\{\begin{array}{l}
1, \text { if there is a } k \text {-type flow } \\
\text { on the destination } i j \\
0, \text { otherwise. }
\end{array}\right.
$$

If $\sum_{k} \xi_{i j}^{k}=2$, the destination $i j$ corresponds to the mixed passenger train infrastructure. If $\sum_{k} \xi_{i j}^{k}=1$ at $\xi_{i j}^{k=1}=1, \xi_{i j}^{k=2}=0$, the destination $i j$ corresponds to the dedicated high-speed line, whereas at $\xi_{i j}^{k=1}=0, \quad \xi_{i j}^{k=2}=1$ it is the conventional passenger train line. If $\sum_{k} \xi_{i j}^{k}=0$, there is no passenger traffic on the route. Each infrastructure type corresponds to the established specific expenditures per passenger, evaluating the time a passenger spends in $k$-type trains, comfortable factor, capital and operational expenditures, $C_{i j}^{k, \sum_{k} \xi_{i j}^{k}}, \mathrm{UAH}$.

In order to formalize the process of simultaneous search for rail passenger network topology and determination of the most probable distribution of high-speed and conventional train flows in the formed network, it has been proposed to use a criterion as the system entropy, adapted to rail passenger-focused transportation. A mathematical model of a search for a rational distribution of passenger train flows is

$H\left(x_{i j}^{k}, \xi_{i j}^{k}\right)=\sum_{i}^{N} \sum_{j}^{M} \sum_{k}^{K} a_{i j}^{k} x_{i j}^{k} \ln \left(\frac{\begin{array}{c}k, \sum_{k j}^{k} \\ p_{i j}^{k}\end{array}}{a_{i j}^{k} x_{i j}^{k}}\right) \rightarrow \max$,

with limitations on passenger flow distribution:

$$
\begin{aligned}
& \sum_{j=1}^{M} \sum_{k=1}^{K} \xi_{i j}^{k} a_{i j}^{k} x_{i j}^{k}=A_{i}^{\text {depart }}, \forall j=1, M, \\
& \sum_{i=1}^{N} \sum_{k=1}^{K} \xi_{i j}^{k} a_{i j}^{k} x_{i j}^{k}=A_{j}^{\text {arriv }}, \forall i=1, N, \\
& \sum_{k} f_{s t, i}^{k}=A_{i}^{\text {depart }}, \sum_{k} f_{s t, j}^{k}=A_{j}^{\text {arriv }},
\end{aligned}
$$

and limitations on the carrying capacity of rail sections

$\sum_{i=1}^{N} \sum_{k=1}^{K} \xi_{i j}^{k} x_{i j}^{k} \leq N_{i j}^{\max k}$

$\sum_{j=1}^{M} \sum_{k=1}^{K} \xi_{i j}^{k} x_{i j}^{k} \leq N_{j i}^{\max k}$

where $p_{i j}^{k, \sum_{k} \xi_{i j}^{k}}$ - is the probability that a $k$-type train will run between points connected by the route $i j$ in the network. The value of $p_{i j}^{k, \sum_{k j} \xi^{k}}$ depends on the expenditures $C_{i j}^{k, \sum_{k}^{k} \xi_{j j}^{k}}$ and is presented as a probability distribution function which monotonically decreases when the expenditures grow and is statistically defined; $a_{i j}^{k}-$ is the capacity of a $k$-type train of the $i j$ destination; $M$ is the multiplicity of arrival stations; $N$ is the multiplicity of departure stations $A_{i}^{\text {depart }}, A_{j}^{\text {arriv }}$ - are total volumes of departures and arrivals, passengers; $N_{i j}^{\max k}, N_{j i}^{\max k}$ - are the maximum number of $k$-type passenger trains, which can be handled along a section in both (odd and even) directions (double track sections); $f_{s t}^{k}-$ is the forecast volumes of passenger flows for every departure point $S$ and destination point $t$ for the passengers intending to travel in a $k$-type train.

\section{Solution to the Mathematical Model on the Base of a Bacterial Foraging Optimization Method}

In order to solve a nonlinear optimization model of mathematical programming (2-7) the study proposes an optimization method based on Bacterial Foraging Optimization (BFO) [10]. On implementing the method the procedure, which helps consider features of the multi-stage task of train flow distribution in a rail network, has been modified, namely, determination of the network topology and distribution of passenger and train flows in the network. To this effect it has been proposed to present each bacteria (agent) in the search space as a set of the parameters of the model considering limitations on their ranges and reducing them to the numerical vector $X^{h}$, consisting of three parts

$X^{h}=\left(C^{1}, C^{2}, C^{3}\right), h=\overline{1, S}$

where $h$ is the number of bacteria in the population $S, h=1, \ldots, S ; \mathrm{S}$ is the number of agents. The first component $C^{3}=(\underbrace{x_{1}, x_{2}, \ldots, x_{q}}_{k=1} \underbrace{x_{1}, x_{2}, \ldots, x_{q}}_{k=2})$ simulates the network's parameters $c_{q}=\xi_{i j}^{k}$ for each train category, $c_{q}=\{1 ; 0\}, q$ is the total number or edges $e_{i j}$. The second component $C^{2}=(b_{1}, b_{2} b_{3}, b_{4}, \ldots, \underbrace{b_{u-1}, b_{u}}_{w=1}$, realizes coefficients which allow determining distribution of parts of the passenger flow $f_{s t}^{k}$ between the two shortest routes obtained, $b_{u}=[0 ; 1] ; W$ is the total number of specified passenger flows $f_{s t}^{k}, w \in W$. In order to determine part of the flow directing along each route it has been proposed to normalize the coefficient by the formula $\frac{b_{1}}{\sum b_{1}+b_{2}}$ for the first route and $\frac{b_{2}}{\sum b_{1}+b_{2}}$ for the second route (Fig. 1) 


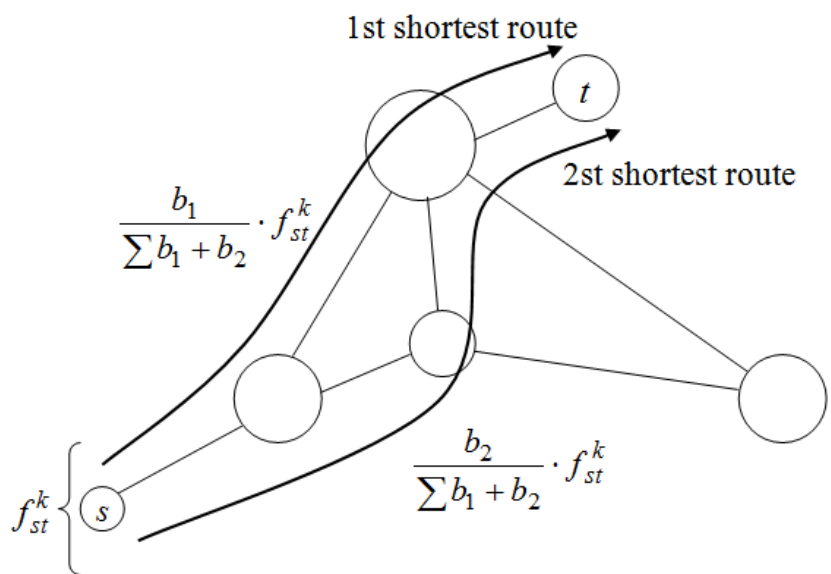

Fig. 1: Diagram of determination of passenger flow distribution by the two shortest routes.

After decoding the values of passenger flows on the base of coefficient normalization, the matrix is filled out; it defines the value of flows on each edge $e_{i j}$ of the graph $G$ (Fig.1).

The third part $C^{3}=(\underbrace{x_{1}, x_{2}, \ldots, x_{q}}_{k=1} \underbrace{x_{1}, x_{2}, \ldots, x_{q}}_{k=2})$ realizes parameters of the train flow intensity $x_{i j}^{k}$ for each destination $q=\{i, j\}$, where $N_{\max }^{k}$ is the maximum possible value of the number of $k$-type passenger trains on the rail line $q$.

Owing to the BFO method, the task assigned has been solved in several stages (Fig. 2). Thus, on the first stage after the determination of a bacterium's position, i.e. finding the numerical vector $X^{h}$, the first part $C^{1}$ of the vector is being decoded, its parameters make it possible to simulate the infrastructure type and evaluate how much is the cost of passenger-kilometers $v^{k}$ which corresponds to a $k$-type train run along the section. On the second stage the passenger routes, which satisfy forecasts of passenger demands for transportation from the departure point to the destination point on the network topology obtained, are being searched. To solve the task it has been proposed to use the well known Yen's and Dijkstra's algorithms [11]. The selection criterion for the shortest path is the minimized total cost of passenger-kilometers with consideration of the infrastructure type on each section included in the route. After calculating the train flow routes, the graph's edges included in the train routes obtained are compared with the appropriate values of the train flow intensity $x_{i j}^{k}$ on each section $i j$ simulated in the part $C^{3}$ of the vector $X^{h}$. Thus, train flow distribution with consideration of various infrastructures in the network is conducted, and, as a result, the costs of flows differ.

On obtaining the alternative development of the rail network topology for express and high-speed trains, it has been proposed to evaluate it with the target function $J\left(X^{h}\right)$, which transforms the task (2)-(8) into an unconstrained optimization task. The target function of unconstrained optimization is

$$
\begin{aligned}
& J\left(X^{h}\right)=\sum_{i}^{N} \sum_{j}^{M} \sum_{k}^{K} a_{i j}^{k} x_{i j}^{k} \ln \left(\frac{p_{i j}^{k}}{a_{i j}^{k} x_{i j}^{k}}\right)+ \\
& +\lambda\left(\sum_{\psi=1}^{\phi}\left(h_{\kappa}(x)\right)^{2}\right) \rightarrow \max ,
\end{aligned}
$$

where $\lambda$ is the parameter of penalty function, $\lambda>0 ; h_{k}(x)$ limitations of the task (3-8) reduced to $h_{\kappa}(x)=0$ or $h_{\kappa}(x) \leq 0, \kappa \in \mathrm{K}$.

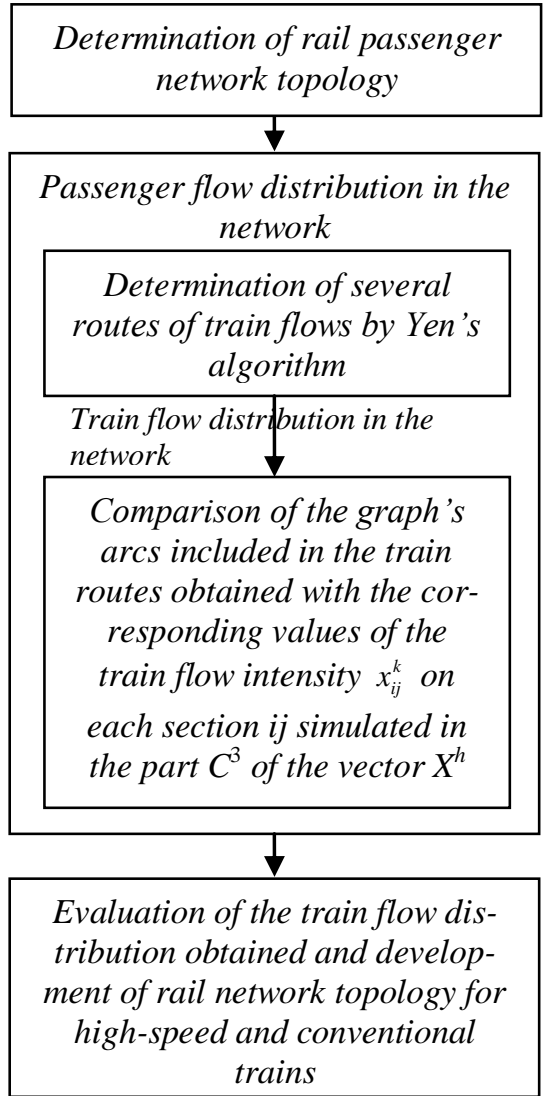

Fig. 2: Sequence of solutions to the task of passenger flow distribution by determining a rational rail network topology for high-speed and conventional trains in the BFO method.

The search procedure for the best solution to the task in the BFO method is based on analogies with the E.Coli bacterium survival in the natural environment [12]. Under such conditions the solution region can be presented as the environment of bacterial clumps with areas of high and low concentrations of useful substances (best and worst values of the target function of the task). The search for the best solution from the bacterium is based on the motility of the bacterium which is based on its function to move linearly (glidingly) and a random change in the movement (rotation) direction space [12].

For modelling the interaction between bacteria the BFO procedure has used the function $J_{a r}\left(X^{h}\right)=\exp \left(\left(M-J\left(X^{h}\right)\right) J_{c c}\left(X^{h}\right)\right)$, where $M$ is the adjustable parameter; $J_{C C}\left(X^{h}\right)$ is the function modelling a signal between cells based on calculation of the amount of attractant (useful substance for cells) and amount of repellent (harmful substance) and is presented as follows

$J_{c c}\left(X^{h}\right)=\sum_{i=1}^{S} J_{c c}^{i}=\sum_{i=1}^{S}\left[-d_{\text {attract }} \exp \left(-w_{\text {attract }} \times\right.\right.$

$\left.\left.\times \sum_{j=1}^{p}\left(X_{j}-X_{j}^{i}\right)^{2}\right]\right)+\sum_{i=1}^{S}\left[h_{\text {repellent }} \times\right.$

$\left.\times \exp \left(-w_{\text {repellent }} \sum_{j=1}^{p}\left(X_{j}-X_{j}^{i}\right)^{2}\right)\right]$,

where $X$ is the bacterium's position in the search space, $R^{p}, X \in R^{p}$, $d_{\text {attract }}$ is the depth of the agent's attractant; $w_{\text {attract }}$ is the measure of width of attractant; $h_{\text {repellent }}$ is the depth of the agent's repellent; $w_{\text {repellent }}$ is the measure of width of repellent. In the framework of software implementation the bacteria were not food for one another, they eliminated one another due to local consumption.

According to the environment utility the cells duplicate or are eliminated from the calculation. Under such conditions in order to 
find an optimal solution to the task the calculation of the evaluation function for the cell utility on each step of chemotaxis, reproduction and elimination is conducted by the formula

$J\left(X^{h}\right)=J\left(X^{h}\right)+J_{a r}\left(X^{h}\right)$. Besides the target function calculation, the BFO method used transfer and gliding operations to change the position of cells in the search area. If one takes that $X^{h}(k, l, n)$ describes the position of each element of the population of bacteria at a $k$-step of chemotaxis, an $l$-step of reproduction and an $n$-event of elimination/dispersal, the transfer is calculated by the formula [13]

$$
X^{h}(k+1, l, n)=X^{h}(k, l, n)+C(h) \varphi(k)
$$

where $C(h)$ is the step size in a certain direction, which allows modelling the process of rotation; $\varphi(k)$ is the vector of random numbers, $\varphi(k) \in R^{p}$.

The gliding operation is being realized by the condition: if in the position $X^{h}(k+1, l, n)$ the value $J(k+1, l, n)$ is better than in the position $X^{h}(k, l, n)$, the following step is being done with the same vector $\varphi$ and in the same direction, and such a repetition can occur $N_{S}$ times. If the condition $J\left(X^{h}\right)(k+1, l, n)<J\left(X^{h}\right)(k, l, n)$ is not fulfilled, so $\varphi$ is re-calculated. All the steps are presented with more detail in [12].

\subsection{Results of the Calculation}

An example of behavior of the mathematical model based on the BFO method is implemented in Scilab [14]. Fig. 3 demonstrates results of the calculation of a rational network topology of highspeed passenger transportation for 18 arbitrarily chosen graph nodes $\mathrm{G}(\mathrm{P}, \mathrm{E})$ (heavy lines stand for dedicated high-speed lines, thin lines stand for conventional passenger lines).

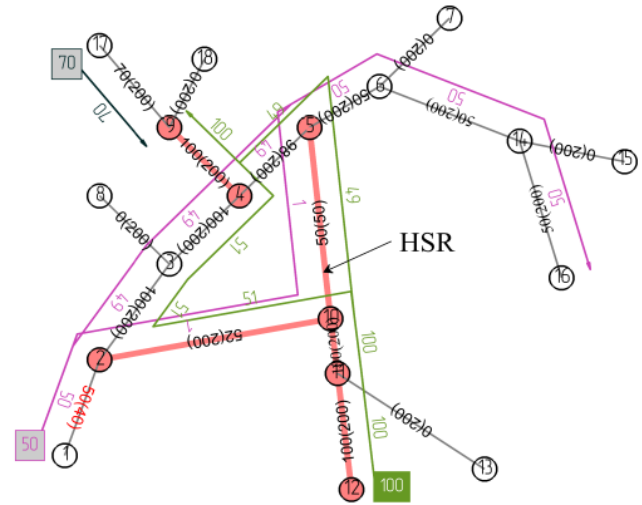

Fig. 3: Results of the calculation of the rational network topology of highspeed passenger transportation for 18 arbitrarily chosen graph nodes.

The diagram of the target function change against the number of iterations in the BFO algorithm is presented in Fig. 4.

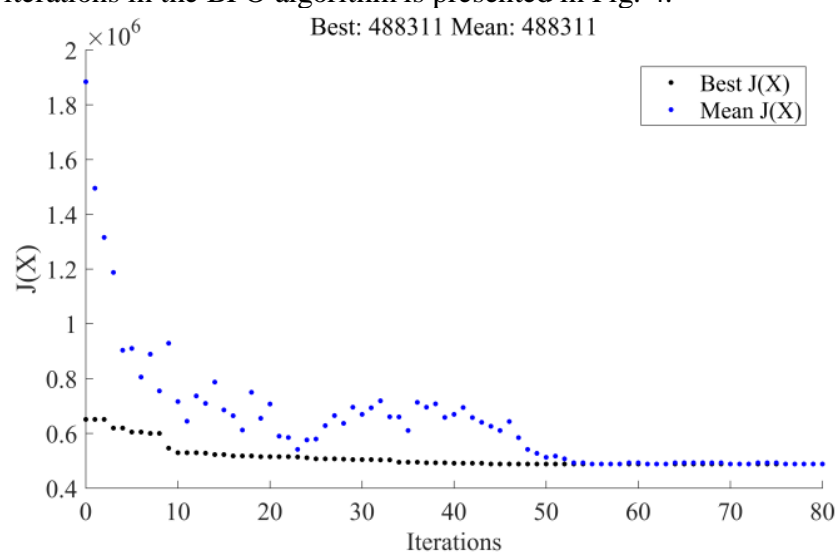

Fig. 4: The target function change against the number of iterations is the BFO method in search for a rational high-speed passenger network topology.
The results of modelling proved high accuracy and speed in the search for a rational rail network topology and distribution of high-speed and conventional trains taking into account the demand for transportation.

\section{Conclusions}

In this section you should present the conclusion of the paper. Conclusions must focus on the novelty and exceptional results you acquired. Allow a sufficient space in the article for conclusions. Do not repeat the contents of Introduction or the Abstract. Focus on the essential things of your article. As isomorphism exists in systems for investigation into the self-organizing train flow distribution process, it is reasonable to use the entropy concept which makes it possible to research and implement connection between micro- and macro levels in the passenger transportation system. On the basis of the second law of thermodynamics, the study proved that the rail passenger transportation system can be presented as a closed physical system tending to a steady equilibrium state, when uncertainty, measured by entropy, is maximal.

In order to solve the task of a rational passenger train flow distribution in the network, an optimization criterion, adopted to rail passenger-oriented transportation conditions has been created in the form of the system entropy.

An entropy model based on the multi-agent optimization methods, namely BFO, has been proposed to formalize the high-speed train distribution process in the network. Unlike existing ones, the model helps simultaneously determine the rail passenger transportation topology and the most probable distribution of high-speed and conventional train flows in the network, which can occur in a real transport system, taking into account the patterns of passenger collective behavior.

The results of modelling have proved high accuracy and speed of the approach. While applying the method of bacteria transfer the procedure which allows considering features of a multi-stage task of train flow distribution in a rail network has been modified, namely, the determination of the network topology and distribution of passenger and train flows.

The mathematic model designed on the base of BFO helps determine the most promising variants of passenger train distribution in the network with minimal capital expenditures for the network modernization and increased economic efficiency of high-speed and conventional train traffic by adapting development of the network topology to the demand for transportation.

\section{Acknowledgement}

This research was supported by Ukrainian State University of Railway Transport (USURT).

\section{References}

[1] Wardrop JC (1952), "Some Theoretical Aspects of Road Traffic Research", Proceedings, Institution of Civil Engineers Part 2, 9, 325-378.

[2] Drezner Z \& Wesolowsky G (2003), "Network design: Selection and Design of Links and Facility Location", Transportation Research Part A, 37, 241-256.

[3] Garrison WL \& Souleyrette RR (1996), "Transportation, Innovation, and Development: The Companion Innovation Hypothesis", Logistics and Transportation Review, 32, 5-37.

[4] Krugman P. (1996), "The Self-Organizing Economy", Blackwell, Malden, 53-100.

[5] Helbing D, Keltsch J \& Molnár P (1997), ”Modeling the evolution of human trail systems", Nature, 388(6637), 47-50.

[6] Newman M. (2003), "The structure and function of complex networks", SIAM Review, 45, 167-256, https://doi.org/10.1137/S003614450342480

[7] Wang L, Li-min Jia, Yong Qin, Jie Xu \& Wen-ting Mo (2011), ”A two-layer optimization model for high-speed railway line planning", Journal of Zhejiang University-SCIENCE A, 12(12), 902-912. 
[8] Butko T, Prokhorchenko A \& Zhurba O (2010), "Modelling of distribution of passenger traffic on trains on the basis of swarm intelligence", Eastern-European Journal of Enterprise Technologies, 2/4(44), 44-47. available online: http://journals.uran.ua/eejet/article/view/2653, last visit:22.05.2018

[9] Xin Qi \& Jian Xiong, "Optimization method of passenger train plan based on stop schedule plan for passenger dedicated line", 2012 2nd International Conference on Uncertainty Reasoning and Knowledge Engineering, IEEE, (2012), pp: 204-207, http://dx.doi.org/10.1109/URKE.2012.6319546

[10] Wilson AJ (1969), "Entropy maximizing models in the theory of trip distributions, mode split and route split", J. Transp. Econ. Policy, 3, 108-126.

[11] Yen JY (1971), "Finding the K shortest loopless path in a network", Management Science, 17, 712-716.

[12] Liu Y \& Passino KM, (2002), "Biomimicry of Social Foraging Bacteria for Distributed Optimization: Models, Principles, and Emergent Behaviors", Journal of Optimization Theory and Applications, 3(115), 603-628, https://doi.org/10.1023/A:1021207331209

[13] Passino KM (2002), "Biomimicry of bacterial foraging for distributed optimization and control", IEEE Control System Magazine, 3(22), 52-67.

[14] Stephen L Campbell, Jean-Philippe Chancelier \& Ramine Nikoukhah, Modeling and Simulation in Scilab/Scicos. New York: Springer, (2006) 